\title{
Energy Partition in Four Confined Circular-Ribbon Flares
}

\author{
Z.M. Cai ${ }^{1,2}$. Q.M. Zhang ${ }^{1,2,3} \cdot$ Z.J. Ning ${ }^{1,2}$. \\ Y.N. $\mathrm{Su}^{1,2} \cdot$ H.S. $\mathrm{Ji}^{1,2}$ \\ (C) Springer $\bullet \bullet \bullet$
}

\begin{abstract}
In this study, we investigated the energy partition of four confined circular-ribbon flares (CRFs) near the solar disk center, which are observed simultaneously by SDO, GOES, and RHESSI. We calculated different energy components, including the radiative outputs in $1-8,1-70$, and $70-370 \AA$, total radiative loss, peak thermal energy derived from GOES and RHESSI, nonthermal energy in flare-accelerated electrons, and magnetic free energy before flares. It is found that the energy components increase systematically with the flare class, indicating that more energies are involved in larger flares. The magnetic free energies are larger than the nonthermal energies and radiative outputs of flares, which is consistent with the magnetic nature of flares. The ratio $\frac{E_{n t h}}{E_{m a g}}$ of the four flares, being 0.70-0.76, is considerably higher than that of eruptive flares. Hence, this ratio may serve as an important factor that discriminates confined and eruptive flares. The nonthermal energies are sufficient to provide the heating requirements including the peak thermal energy and radiative loss. Our findings impose constraint on theoretical models of confined CRFs and have potential implication for the space weather forecast.
\end{abstract}

Keywords: Flares, Dynamics; Flares, Energetic Particles; Heating, in Flares; Magnetic fields, Corona

\footnotetext{
$凶$ Q.M. Zhang

zhangqm@pmo.ac.cn

1 Key Laboratory of Dark Matter and Space Astronomy, Purple Mountain Observatory, CAS, Nanjing 210023, China

2 School of Astronomy and Space Science, University of Science and Technology of China, Hefei 230026, China

3 CAS Key Laboratory of Solar Activity, National Astronomical Observatories, CAS, Beijing 100101, China
} 


\section{Introduction}

Solar flares and coronal mass ejections (CMEs) are the most energetic activities in the solar system, which are considered as the main source of space weather (Fletcher et al., 2011; Webb and Howard, 2012; Gopalswamy, 2016; Patsourakos et al., 2020). The accumulated magnetic free energy $\left(10^{29}-10^{33} \mathrm{erg}\right)$ in active regions (ARs) is released within a short period of time via magnetic reconnection (e.g., Antiochos, DeVore, and Klimchuk, 1999; Amari et al., 2000; Chen \& Shibata, 2000; Lin and Forbes, 2000; Moore et al., 2001). The released energy goes into the thermal energy of localized hot plasmas, kinetic energy of reconnection outflows, kinetic energy of CMEs, nonthermal energies of the accelerated electrons and/or ions, and radiations from radio to hard X-ray (HXR) and even $\gamma$-ray wavelengths (Forbes and Acton, 1996; Stoiser et al., 2007; Kretzschmar et al., 2010; Milligan et al., 2012; Caspi, Krucker, and Lin, 2014; Inglis and Christe, 2014; Warmuth and Mann, 2016a, b). According to their association with CMEs, flares are classified into confined and eruptive types (e.g., Moore et al., 2001; Cheng et al., 2011; Su et al., 2011, 2015; Veronig and Polanec, 2015; Li et al., 2020; Kliem et al., 2021). A large number of confined flares result from failed filament eruptions due to the strong confinement of the overlying field (Ji et al., 2003; Liu et al., 2014; Sun et al., 2015; Zhang et al., 2015; Yang and Zhang, 2018; Yan et al., 2020). Sometimes, confined flares are triggered by loop-loop interaction ( $\mathrm{Su}$ et al., 2013; Kushwaha et al., 2014; Ning et al., 2018).

Contrary to two-ribbon flares, circular-ribbon flares (CRFs) are a special type of flares that consist of a short, compact inner ribbon and a bright, outer ribbon with a circular or elliptical shape (Masson et al., 2009; Joshi et al., 2015; Liu et al., 2015; Hernandez-Perez et al., 2017; Devi et al., 2020; Kashapova et al., 2020; Prasad et al., 2020; Joshi, Joshi, and Mitra, 2021). The three-dimensional (3D) magnetic configuration of CRFs is usually related to a fan-spine structure associated with a magnetic null point (Wang and Liu, 2012; Zhang et al., 2012; Sun et al., 2013; Hou et al., 2019; Lee et al., 2020; Liu et al., 2020; Yang et al., 2020; Zhang et al., 2021). CRFs are occasionally accompanied by coronal jets or cool surges (Zhang et al., 2016; Li et al., 2017; Xu et al., 2017; Dai et al., 2020; Zhang et al., 2020). The dynamic evolution of CRFs, including magnetic reconnection near the null point, particle acceleration and precipitation, chromospheric evaporation and condensation, are found to resemble those of two-ribbon flares (Zhang, Li, and Ning, 2016; Zhang, Li, and Huang, 2019).

Till now, comprehensive investigations on energetics of eruptive flares are abundant (Emslie et al., 2004, 2005, 2012; Milligan et al., 2014; Warmuth and Mann, 2016a b). The energy partitions in flares and CMEs are comparable, especially for X-class eruptive flares (Feng et al., 2013). Using multiwavelength observations from the Atmospheric Imaging Assembly (AIA; Lemen et al., 2012) on board the Solar Dynamics Observatory (SDO), the energetics of nearly 400 eruptive flares were studied in detail (Aschwanden, Xu and Jing, 2014; Aschwanden et al., 2015, 2016, 2017). However, the investigation on energy partition in confined flares is rare. Thalmann et al. (2015) studied an X1.6 flare in AR 12192 on 2014 October 22 . The nonthermal energy $\left(\sim 1.6 \times 10^{32} \mathrm{erg}\right)$ in flareaccelerated electrons is found to account for $\sim 10 \%$ of the free magnetic energy 
Table 1. Information on the four flares in our study. Here, $t_{s t a}, t_{\text {peak }}$, and $t_{\text {end }}$ represent the start, peak, and end times of the flares in GOES 1-8 Å, respectively. $\Delta t$ denotes the rough lifetime.

\begin{tabular}{lcccccccc}
\hline Flare & Date & $\begin{array}{c}t_{\text {sta }} \\
(\mathrm{UT})\end{array}$ & $\begin{array}{c}t_{\text {peak }} \\
(\mathrm{UT})\end{array}$ & $\begin{array}{c}t_{\text {end }} \\
(\mathrm{UT})\end{array}$ & $\begin{array}{c}\Delta t \\
(\mathrm{~min})\end{array}$ & AR & Location & Class \\
\hline CRF1 & 10-May-2012 & $20: 20$ & $20: 26$ & $21: 20$ & 60 & 11476 & N12E12 & M1.7 \\
CRF2 & 07-Nov-2013 & $03: 34$ & $03: 39$ & $03: 54$ & 20 & 11890 & S13E28 & M2.3 \\
CRF3 & 29-Dec-2013 & $14: 38$ & $14: 45$ & $15: 10$ & 32 & 11936 & S16W05 & C5.1 \\
CRF4 & 05-Mar-2014 & $00: 10$ & $00: 15$ & $00: 25$ & 15 & 11991 & S27W07 & C4.8 \\
\hline
\end{tabular}

before flare. Kushwaha et al. (2015) studied an M6.2 flare in AR 10646 on 2004 July 14. The time evolution of thermal energy is found to show a good correlation with the variations in cumulative nonthermal energy, validating the well-known Neupert effect in confined flares. Zhang et al. (2019) explored various energy components in two homologous confined CRFs of the same class (M1.1) in AR 12434 , including the peak thermal energy, nonthermal energy in electrons, total radiative loss of hot plasma, and radiative output in $1-8 \AA$ and $1-70 \AA$. The two flares have similar energy partition, and the nonthermal energy is sufficient to provide the heating requirement incorporating the peak thermal energy and radiative loss.

In this study, we selected four confined CRFs near the solar disk center observed by SDO/AIA (Song and Tian, 2018). In Section 2 we briefly describe the data sets and calibration. We are not interested in the triggering mechanism of each flare, which has been extensively studied. We focus on the estimation of various energy components of flares in Section 3. The results are compared with previous findings in Section 4 Finally, a summary is given in Section 5

\section{Data Sets and Calibration}

The date/time, location, and GOES class of the four flares (CRF1, CRF2, CRF3, and CRF4) are listed in Table 1 Among the four flares, two are M-class and the others are C-class. The flares were observed by SDO/AIA in extreme-ultraviolet (EUV) wavelengths $(131,171$, and $304 \AA$ ). The AIA level_1 data were calibrated using the standard Solar Software (SSW) program aia_prep.pro. In Figure 1, the $171 \AA$ images illustrate the whole evolution of the flares (see also the online movies). The four flares share a basic similarity in evolution. The inner ribbons and part of the outer ribbons brightened first. Then, the rest of the outer ribbons brightened sequentially, which is consistent with previous findings ( $\mathrm{Li}$ et al.,2017; $\mathrm{Xu}$ et al., 2017). Finally, the brightness of flare ribbons declined gradually with time and died out. It is noted that CRF1 was associated with a blowout coronal jet propagating in the northwest direction, while the remaining three flares were not associated with jets.

The line-of-sight (LOS) and vector magnetograms of the photosphere were observed by the Helioseismic and Magnetic Imager (HMI; Scherrer et al., 2012) 

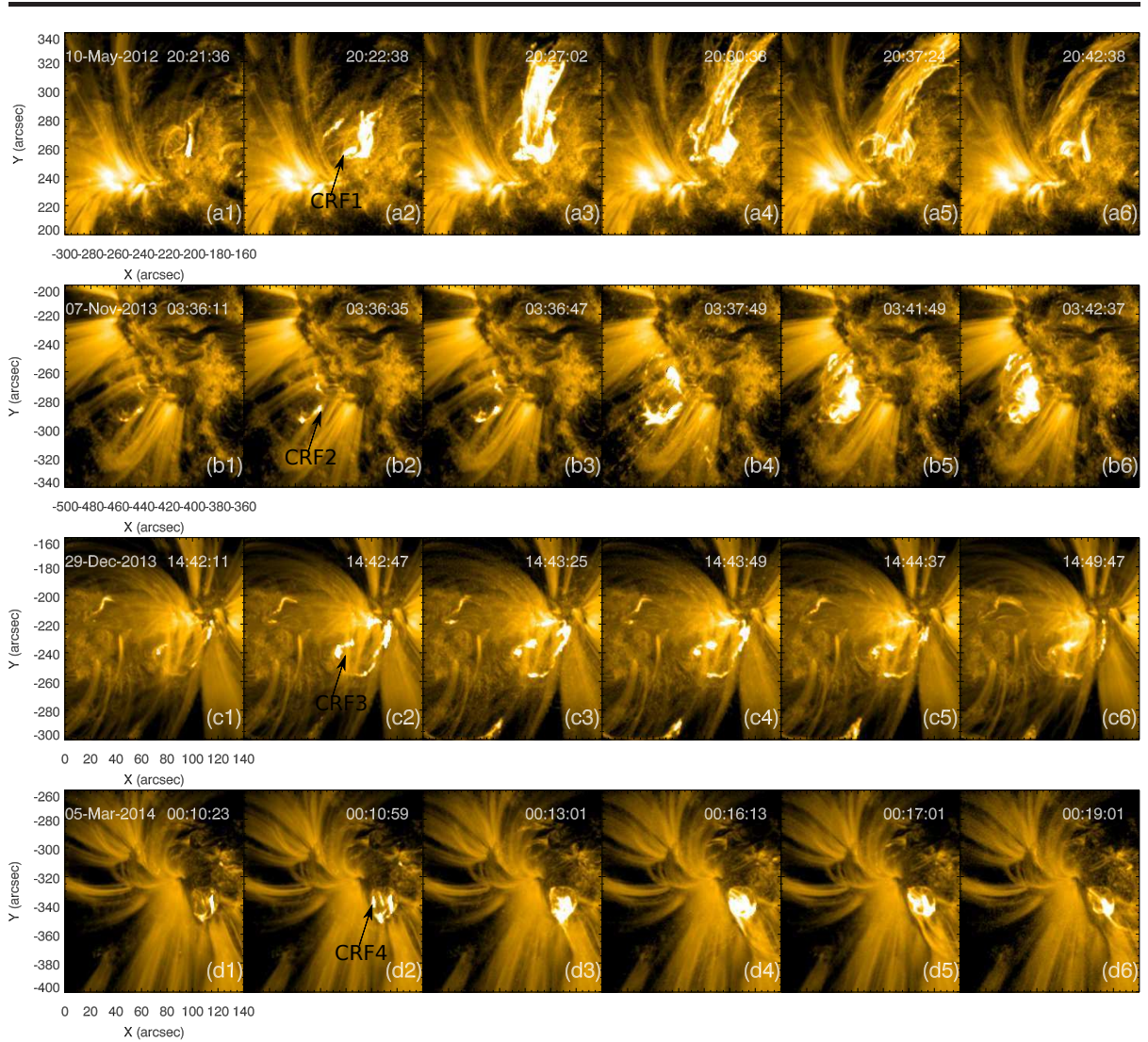

Figure 1. Snapshots of the four flares in AIA $171 \AA$. The top row is for CRF1 and the bottom row is for CRF4. (Four animations of this figure are available online.)

on board SDO with cadences of $45 \mathrm{~s}$ and $720 \mathrm{~s}$, respectively. The HMI level_1 data were calibrated using the SSW program hmi_prep. pro. The pre-flare vector magnetograms were used to extrapolate the coronal magnetic field. Figure 2 shows vector magetograms of the four ARs hosting the flares. We carried out potential field extrapolation based on the LOS magnetograms using the Green's function method (Chiu and Hilton, 1977; Seehafer, 1978). To carry out the nonlinear force-free field (NLFFF) modeling, we use vector magnetograms and the "optimization" method (Wiegelmann, Inhester and Sakurai, 2006; Wiegelmann et al., 2008). Pre-processing of the photospheric magnetograms is conducted before NLFFF extrapolation. Figure 3 shows the nonpotential magnetic configurations (blue lines) of the four flares. The bottoms of the boxes are EUV $304 \AA$ images of the corresponding flares. It is clear that the magnetic configurations of the four flares are dome-like, implying the existence of well-known fan-spine topology (e.g., Sun et al., 2013; Zhang et al., 2021). For CRF1, the direction of possible spine line is consistent with the axis of the blowout jet (panel (a)). Besides, the footpoints of field lines match the ribbons of the flares well, thus validating the reliability of NLFFF extrapolation. 

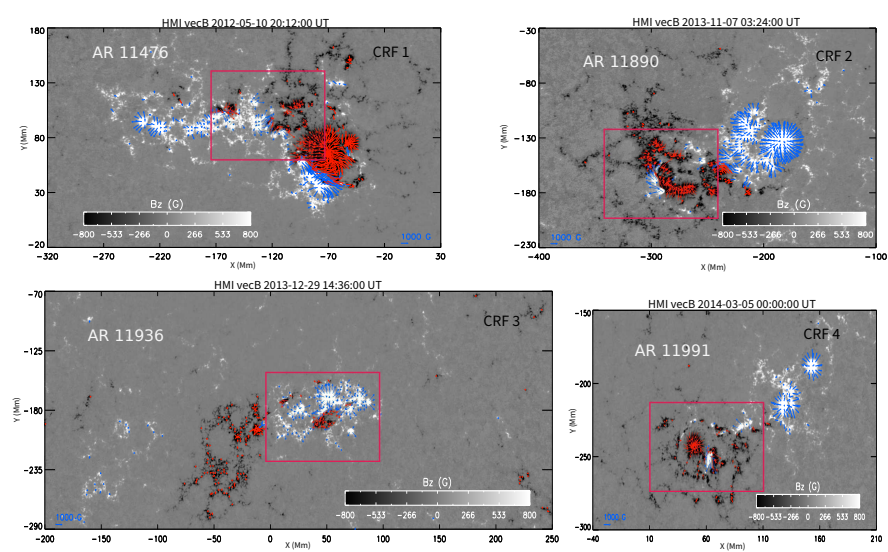

Figure 2. HMI vector magetograms of the four ARs hosting the flares. NLFFF extrapolations were performed using these magnetograms. The red boxes indicate regions for calculating the magnetic free energy, where flares took place.

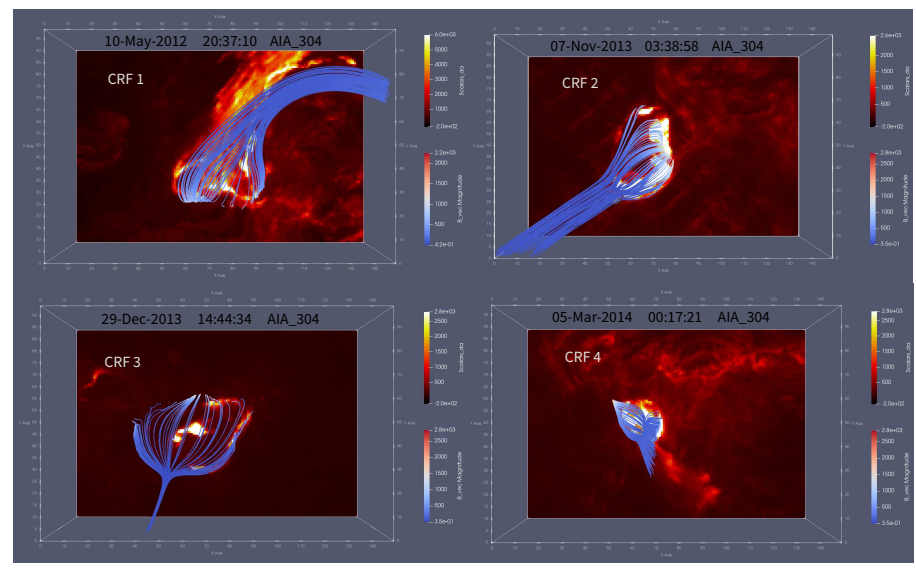

Figure 3. Nonpotential magnetic configuration of the four flares (blue lines). The bottoms of the boxes are AIA $304 \AA$ images of the flares.

The solar irradiance from a broad band ranging from $1-70 \AA$ was directly measured by the EUV SpectroPhotometer (ESP) belonging to the Extreme Ultraviolet Variability Experiment (EVE; Woods et al., 2012) on board SDO. The Multiple EUV Grating Spectrographs (MEGS)-A on board EVE, covering the 6-37 $\mathrm{nm}$ range, records a complete spectrum with a time cadence of $10 \mathrm{~s}$ and a spectra resolution of $1 \AA$. The standard SSW program eve_integrate_line.pro was employed to integrate irradiance over $70-370 \AA$ using the EVS spectral data from MEGS-A. The soft X-ray (SXR) fluxes of the flares in 1-8 $\AA$ were recorded by the GOES spacecraft. The isothermal temperature $\left(T_{e}\right)$ and emission measure (EM) of the SXR-emitting plasma were derived from the ratio of GOES fluxes (White, Thomas, and Schwartz,2005). The HXR fluxes at different energy bands were obtained from the Ramaty Hight Energy Solar Spectroscopic Imager (RHESSI; Lin et al., 2002). We made HXR images using the CLEAN method 
Table 2. Description of the observational parameters.

\begin{tabular}{cccc}
\hline Instrument & $\begin{array}{c}\lambda \\
(\AA)\end{array}$ & $\begin{array}{c}\text { Cadence } \\
(\mathrm{s})\end{array}$ & $\begin{array}{c}\text { Pixel Size } \\
\left({ }^{\prime \prime}\right)\end{array}$ \\
\hline SDO/AIA & $131,171,304$ & 12 & 0.6 \\
SDO/HMI & 6173 & 45,720 & 0.6 \\
SDO/EVE & $1-70$ & 0.25 & $\ldots$ \\
SDO/EVE & $70-370$ & 10 & $\ldots$ \\
GOES & $1-8$ & 2.05 & $\ldots$ \\
RHESSI & $3-50 \mathrm{keV}$ & 4.0 & 4.0 \\
\hline
\end{tabular}

(Hurford et al., 2002) at energy bands of 3-6 and 6-12 keV. The observational properties of the instruments are listed in Table 2 .

\section{Energy Partition}

Using multiwavelength observations, we calculated different energy components, including: (i) radiative outputs in $1-8 \AA, 1-70 \AA$, and $70-370 \AA$; (ii) radiative loss from the SXR-emitting plasma; (iii) peak thermal energy of the SXR-emitting plasma; (iv) kinetic energy in flare-accelerated electrons; and (v) magnetic free energy.

\subsection{Radiative Outputs}

As described in Feng et al. (2013), the radiative output of a certain waveband $(\lambda)$ is derived by integrating the background-subtracted light curve $\left(f_{\lambda}\right)$,

$$
U_{\lambda}=2 \pi d^{2} \int_{t_{1}}^{t_{2}} f_{\lambda}(t) d t
$$

where $d \approx 1.496 \times 10^{11} \mathrm{~m}(1 \mathrm{AU})$ signifies the distance between the Sun and Earth, $t_{1}$ and $t_{2}$ represent the lower and upper time limits (Zhang et al., 2019).

In Figure 4, the left panels show SXR light curves of the flares in 1-8 $\AA$, with the dashed lines indicating the background fluxes during the flares. The right panels show background-subtracted light curves of the flares. The radiative output $U_{1-8}$ is calculated by integrating the background-subtracted fluxes between the two dashed lines. The values of $U_{1-8}$, falling in the range of $(0.13-1.63) \times 10^{28}$ erg, are listed in the second column of Table 3 .

Likewise, the left panels of Figures 5.6] show light curves of the four flares in 1-70 $\AA$ and 70-370 $\AA$. The right panels of Figures [5] 6 show backgroundsubtracted light curves of the flares. The radiative outputs $U_{1-70}$ and $U_{70-370}$ are calculated in the same way. The values of $U_{1-70}$, falling in the range of $(2.8-41) \times 10^{29} \mathrm{erg}$, are listed in the third column of Table 3 . The values of $U_{70-370}$, falling in the range of $(1.8-19.0) \times 10^{28} \mathrm{erg}$, are listed in the fourth 

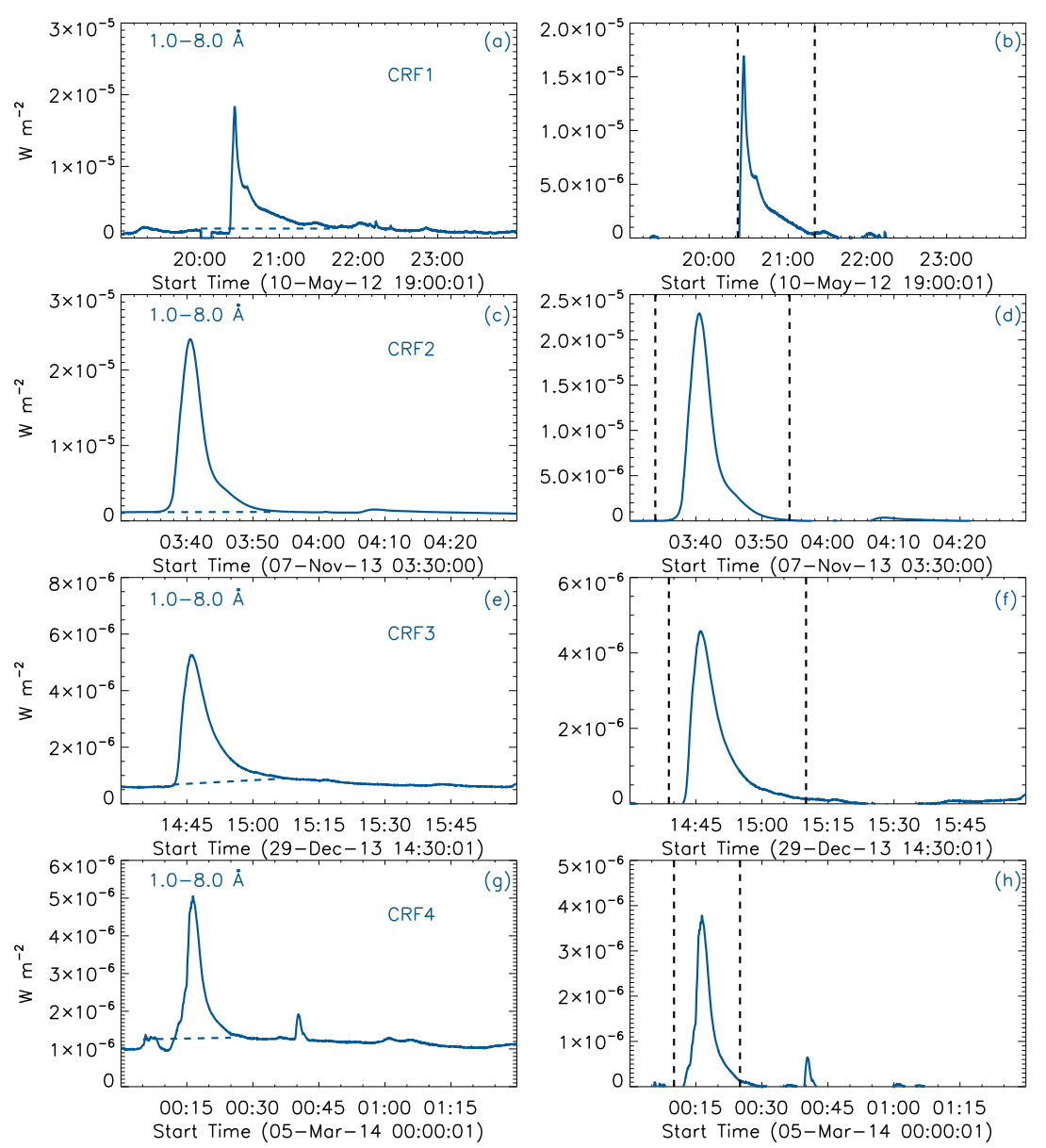

Figure 4. Left panels: SXR light curves of the flares in $1-8 \AA$. The dashed lines indicate the background fluxes during the flares. Right panels: background-subtracted light curves of the flares in 1-8 $\AA$. The vertical dashed lines represent the lower and upper time limits of integrals.

column of Table 3 . It is seen that $U_{1-70}$ is $15-24$ times larger than $U_{70-370}$ and is $\geq 200$ times larger than $U_{1-8}$, which are consistent with previous results for eruptive (Feng et al., 2013) and confined flares (Zhang et al., 2019). The total radiative output $\left(U_{1-370}\right)$ in $1-370 \AA$ of the flares are estimated to be the sum of $U_{1-70}$ and $U_{70-370}$, i.e., $U_{1-370}=U_{1-70}+U_{70-370}$.

\subsection{Radiative Loss from SXR-emitting Plasma}

The total radiative loss from hot plasma emitting SXR can be expressed as:

$$
T_{\text {rad }}=\int_{t_{1}}^{t_{2}} \operatorname{EM}(t) \times \Lambda\left(T_{e}(t)\right) d t
$$


Cai et al.
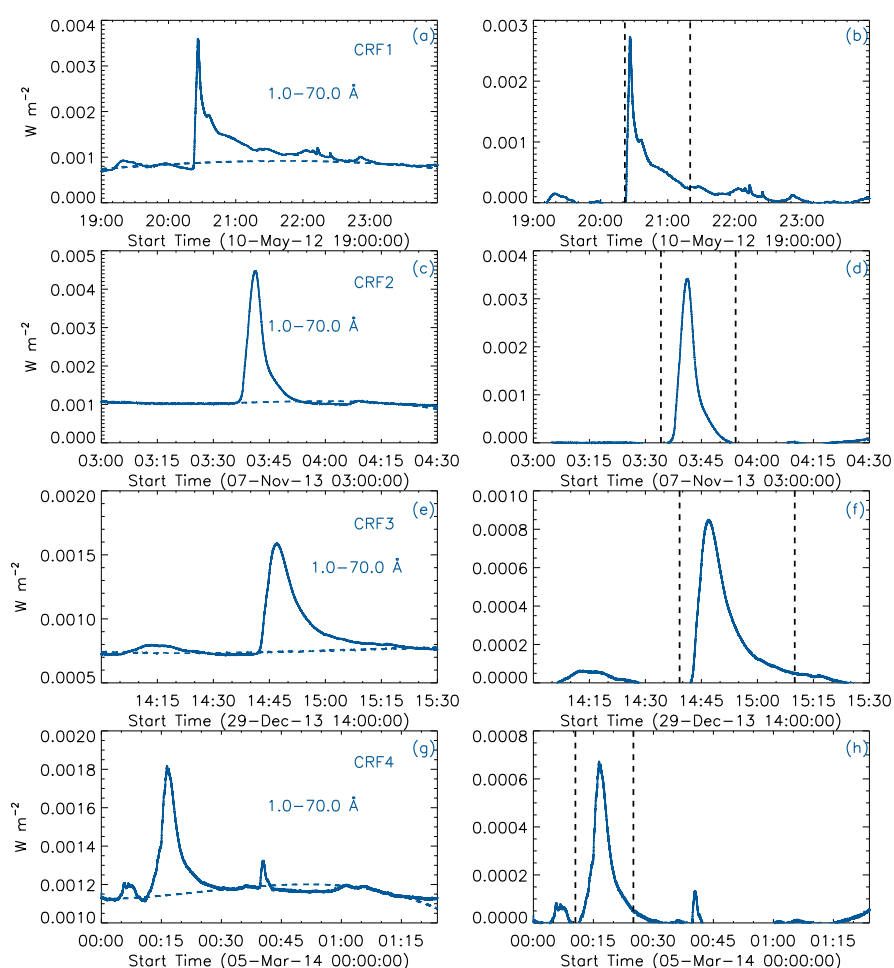

Figure 5. Left panels: light curves of the flares in 1-70 A observed by SDO/EVE. The dashed lines indicate the background fluxes during the flares. Right panels: background-subtracted light curves of the flares in 1-70 $\AA$. The vertical dashed lines represent the lower and upper time limits of integrals.

Table 3. Event List with Component Energies in unit of $10^{29} \mathrm{erg}$.

\begin{tabular}{ccccccccccc}
\hline Flare & $1-8$ & $1-70$ & $70-370$ & $T_{\text {rad }}$ & $E_{t h, G}$ & $E_{t h, R}$ & $\frac{E_{t h, G}}{E_{t h, R}}$ & $E_{n t h}$ & $E_{\text {mag }}$ & $\frac{E_{n t h}}{E_{\text {mag }}}$ \\
\hline CRF1 & 0.163 & 41.0 & 1.90 & 2.9 & 20.00 & 6.26 & 3.2 & 130 & 172.0 & $75.6 \%$ \\
CRF2 & 0.085 & 17.0 & 0.73 & 2.8 & 24.60 & 4.43 & 5.6 & 69 & 97.6 & $70.7 \%$ \\
CRF3 & 0.030 & 6.1 & 0.30 & 0.75 & 5.27 & 3.45 & 1.5 & 19 & 25.2 & $75.4 \%$ \\
CRF4 & 0.013 & 2.8 & 0.18 & 0.86 & 5.34 & 2.88 & 1.9 & 13 & 17.6 & $73.9 \%$ \\
\hline
\end{tabular}

where $\Lambda\left(T_{e}\right)$ denotes the radiative loss rate (Cox and Tucker, 1969), $\operatorname{EM}(t)$ and $T_{e}(t)$ represent the time evolution of EM and $T_{e}$. Figure 7 shows the dependence of $\Lambda$ on $T_{e}$ in the range of $10^{6}-10^{8} \mathrm{~K}$ obtained from CHIANTI 9.0 database by adopting the coronal abundances (Dere et al., 2019).

Figure 8 shows $\operatorname{EM}(t)$ and $T_{e}(t)$ of the four flares derived from GOES observations. The vertical dashed lines indicate $t_{1}$ and $t_{2}$ for integral in Equation 2 . The values of $T_{\text {rad }}$, being $(0.75-2.9) \times 10^{29} \mathrm{erg}$, are listed in the fifth column of Table 3, It is seen that $T_{\text {rad }}$ is several tens of times higher than $U_{1-8}$ (Feng et al., 2013; Zhang et al., 2019). 

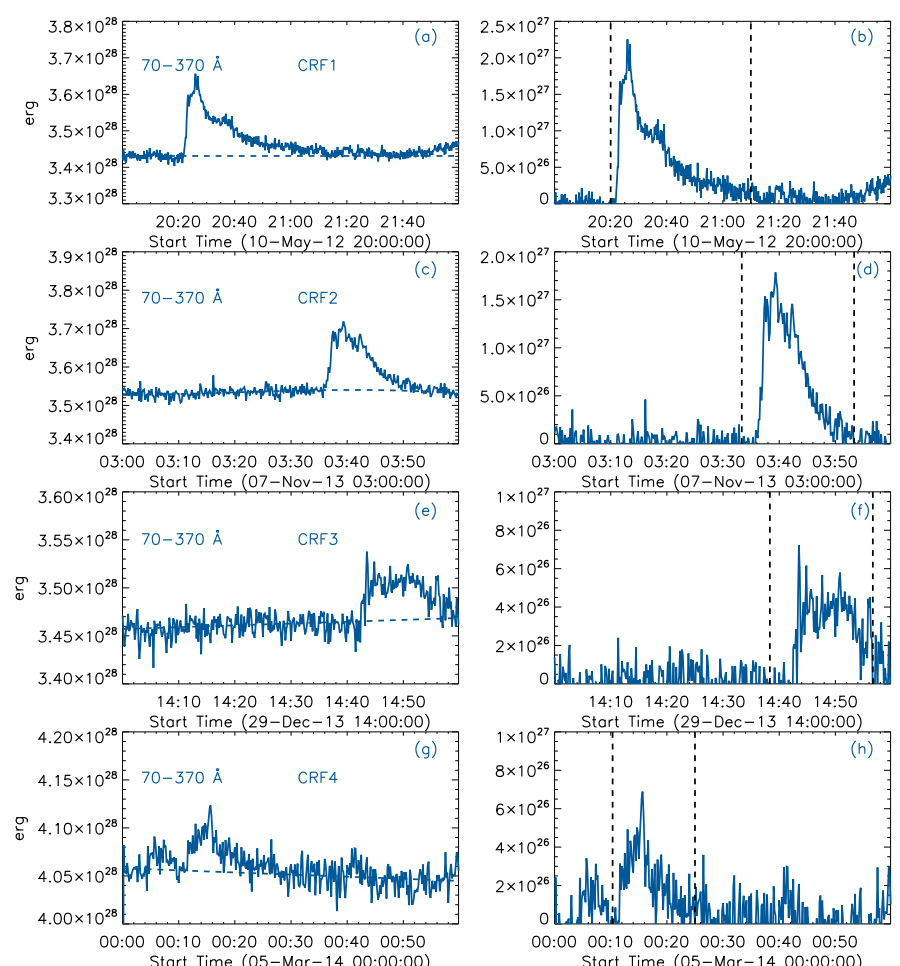

Figure 6. Left panels: light curves of the flares in $70-370 \AA$ observed by SDO/EVE. The dashed lines indicate the background fluxes during the flares. Right panels: background-subtracted light curves of the flares in $70-370 \AA$. The vertical dashed lines represent the lower and upper time limits of integrals.

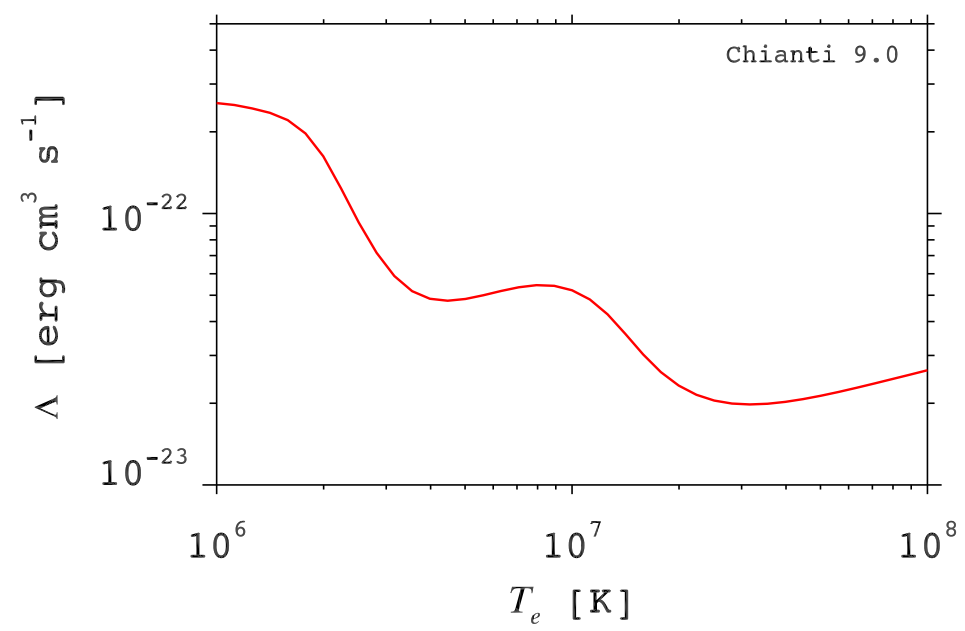

Figure 7. Radiative loss rate $\Lambda\left(T_{e}\right)$ as a function of temperature $\left(T_{e}\right)$ calculated from Chianti 9.0 database. 

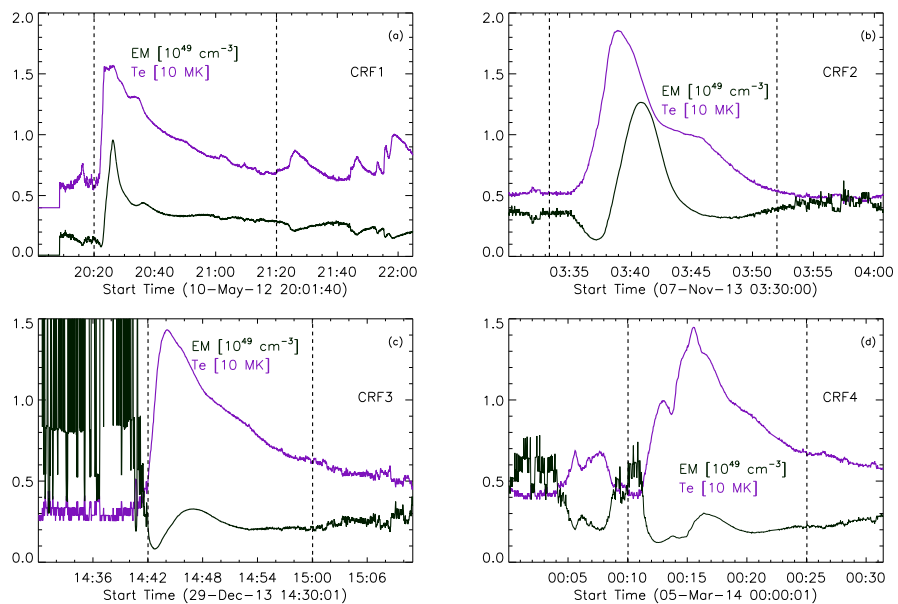

Figure 8. Time evolutions of $T_{e}$ and EM of the four flares obtained from GOES observations.

\subsection{Peak Thermal Energy of SXR-emitting Plasma}

The thermal energy of the hot plasma of flares is expressed as:

$$
E_{t h}=3 n_{e} k_{B} T_{e} f V=3 k_{B} T_{e} \sqrt{\mathrm{EM} \times f V},
$$

where $n_{e}$ is the electron number density, $V$ is the total volume of hot plasma, and $f \approx 1$ denotes the filling factor (Emslie et al., 2012; Warmuth and Mann, $2016 \mathrm{~b})$. In the following, we calculate the peak thermal energy derived from GOES and RHESSI (Warmuth and Mann, 2016b).

For CRFs, whose outer ribbons hardly expand with time, $V \approx A^{3 / 2}$ is assumed to be constant, where $A$ denotes the flare area encircled by the outer ribbons (Zhang et al., 2019). Figure 9] shows $131 \AA$ images of the four flares encircled by the white boxes when their brightness is nearly maximal. AIA $131 \AA$ channel is dominated by the emissions of Fe XXI line $(\log T \approx 7.05)$ during flares (Lemen et al., 2012). In Figure 10, the $131 \AA$ light curves of the flares (blue lines) are compared with the SXR light curves (purple lines), showing that the light curves have good correlations with correlation coefficients of $0.96,0.80,0.94$, and 0.90 , respectively. Therefore, the hot plasma observed in $131 \AA$ serves as a proxy of SXR-emitting plasma. The areas of flares are calculated by summing up the pixels whose intensities are above an ad hoc criterion, which is taken to be $\sim 20$ times higher than the average intensity of the nearby quiet region. The projection effect of $A$ is corrected by multiplying a factor of $(\cos \mu)^{-1}$, where $\mu$ signifies the longitude of flare core. The corresponding $A$ and $V$ in $131 \AA$ are listed in the second and fourth columns of Table 4 . Combining the four flares in this study with the two M1.1 flares in AR 12434, it is found that the thermal source volumes are systematically larger in M-class flares than C-class flares (Warmuth and Mann, 2020).

Equation 3 indicates that the peak thermal energy is reached when $T_{e} \sqrt{\mathrm{EM}}$ is maximal. Using observations from GOES (Figure 8), we calculated the peak 

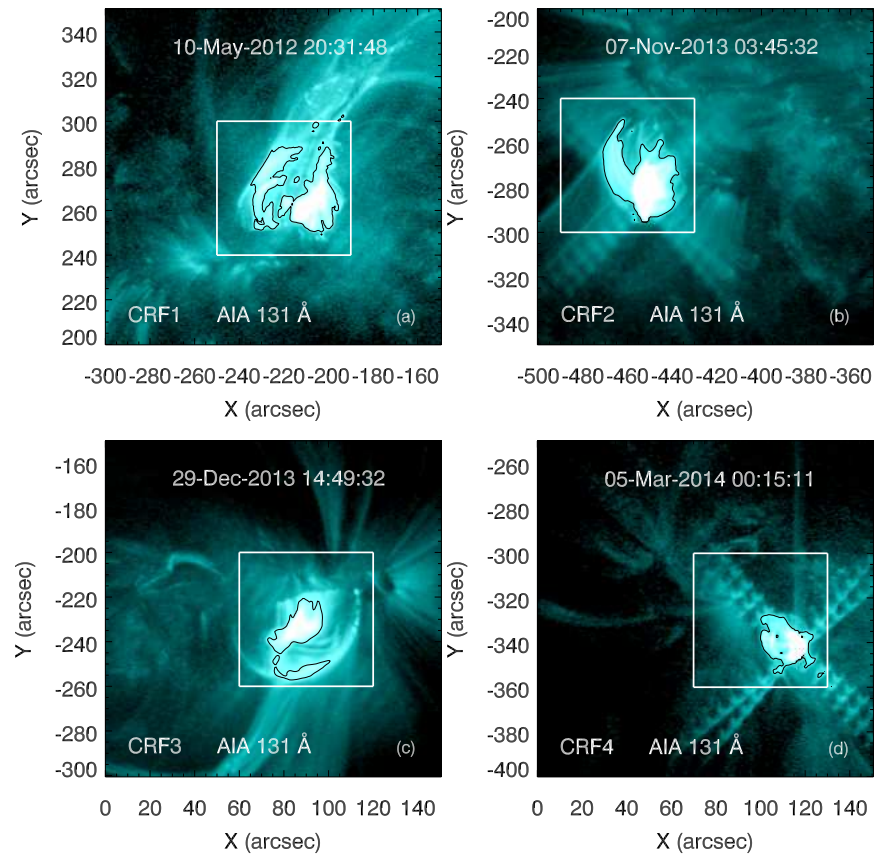

Figure 9. AIA $131 \AA$ images of the four flares near their peak times. Intensity contours of the images are drawn with black solid lines. The white boxes indicate the areas for calculating the light curves in Figure 10

Table 4. Evaluation of the area $\left(\times 10^{18} \mathrm{~cm}^{2}\right)$, volume $\left(\times 10^{28} \mathrm{~cm}^{3}\right)$, and peak thermal energy $\left(\times 10^{29} \mathrm{erg}\right)$ of hot plasma.

\begin{tabular}{ccccccc}
\hline Flare & $A_{131}$ & $A_{\mathrm{HXR}}$ & $V_{131}$ & $V_{\mathrm{HXR}}$ & $E_{t h, G}$ & $E_{t h, R}$ \\
\hline CRF1 & 4.63 & 2.69 & 1.00 & 0.44 & 20.00 & 6.26 \\
CRF2 & 4.86 & 1.78 & 1.07 & 0.24 & 24.60 & 4.43 \\
CRF3 & 2.11 & 2.46 & 0.31 & 0.39 & 5.27 & 3.45 \\
CRF4 & 2.16 & 2.36 & 0.32 & 0.36 & 5.34 & 2.88 \\
\hline
\end{tabular}

values $E_{t h, G}$ of the flares, which are listed in Table 3 and Table 4 . The peak values $E_{t h, G}$ fall in the range of $(5.3-24.6) \times 10^{29} \mathrm{erg}$. The total heating requirements of the flares, including the peak thermal energy and radiative loss, are estimated to be $(0.6-2.7) \times 10^{30} \mathrm{erg}$. Conductive energy loss is not considered in this study since conduction may be severely suppressed or conduction loss is recycled through conduction-driven evaporation (Warmuth and Mann, 2020). Note that CRF1 was accompanied by a blowout jet. The total thermal energy of $\mathrm{CRF} 1$ and the jet is estimated to be $(2.3-2.5) \times 10^{30} \mathrm{erg}$, considering that the 

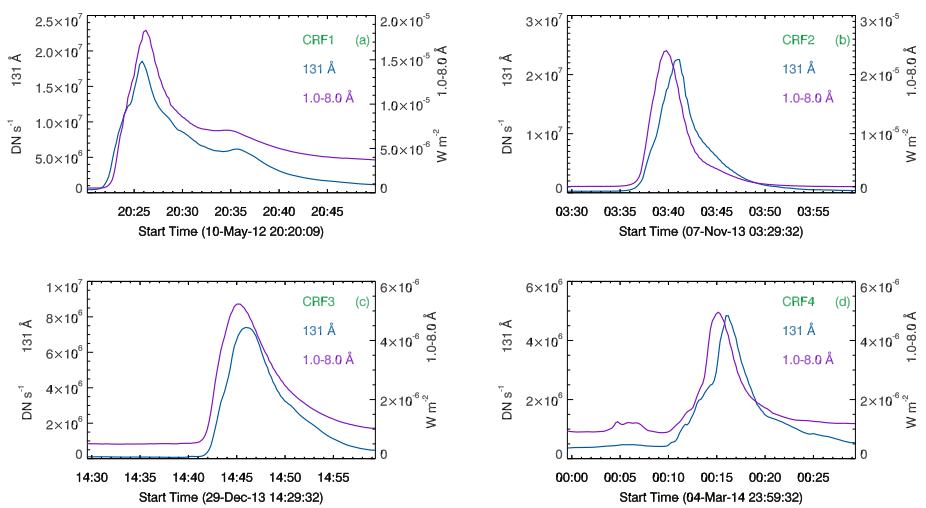

Figure 10. AIA $131 \AA$ light curves (blue lines) and SXR light curves (purple lines) of the four flares.

thermal energies of jets account for $\frac{1}{7}$ to $\frac{1}{4}$ of the footpoint flares (Shimojo and Shibata, 2000).

Figure 11] shows the HXR images of the four flares near the HXR peak times. The energy bands are 6-12 keV for the two M-class flares and 3-6 keV for the two C-class flares. It is clear that the HXR source is single and small for each flare. The contours of $50 \%$ peak intensities are drawn with black lines. The 6-12 $\mathrm{keV}$ sources of the two M-class flares and 3-6 keV sources of the two C-class flares are considered. The area of thermal source observed by RHESSI is taken to be the total area of pixels within the black lines. However, we found that the area and volume are comparable for different energy bands. The corresponding values of $A$ and $V$ are listed in the third and fifth columns of Table 4

Figure 12] shows selected HXR spectra of the four flares obtained from RHESSI observations. The spectra are fitted with a combination of a thermal component and a thick-target nonthermal component. The fitting is performed using the standard SSW program thick2 . pro in the OSPEX package. The parameters of thermal component, including $T$ in unit of MK and EM in unit of $10^{49} \mathrm{~cm}^{-3}$, are labeled. Using Equation 3 the peak thermal energies $\left(E_{t h, R}\right)$ derived from RHESSI are calculated and listed in Table 3 and Table 4 The ratio of $\frac{E_{t h, G}}{E_{t h, R}}$ is accordingly obtained and listed in the eighth column of Table 3 It is revealed that the ratio is greater than 1.0 for all events, which is consistent with previous results (Warmuth and Mann, 2020).

\subsection{Nonthermal Energy in Flare-accelerated Electrons}

To estimate the nonthermal energy in flare-accelerated electrons, we integrated the power of injected electrons over time (Ning and Cao, 2010):

$$
E_{n t h}=\int_{t_{1}}^{t_{2}} P_{n t h}(t) d t=\int_{t_{1}}^{t_{2}} \frac{d E_{n t h}}{d t} d t
$$



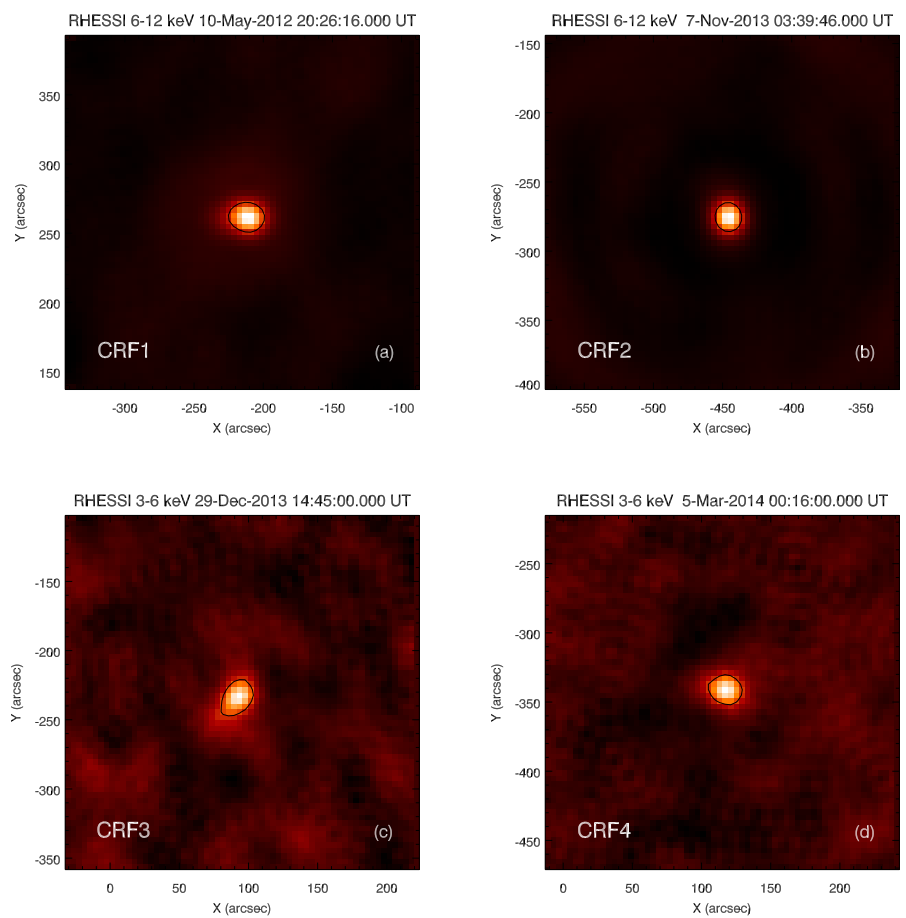

Figure 11. HXR images of the four flares near the HXR peak times. The energy bands are 6-12 keV for the two M-class flares and 3-6 keV for the two C-class flares, respectively. The black lines represent contours of $50 \%$ peak intensities.

where $t_{1}$ and $t_{2}$ represent the start and end times of flare at energy band of 25-50 keV. Here, $P_{n t h}(t)$ can be calculated by integrating the electron powerlaw spectrum above a low-energy cutoff $\left(E_{c}\right)$ and below a high-energy cutoff $\left(E_{h} \approx 30 \mathrm{MeV}\right)$ :

$$
P_{n t h}(t)=\frac{d E_{n t h}}{d t}=\int_{E_{c}}^{E_{h}} A_{0} E_{0}^{-\delta} d E_{0},
$$

where $A_{0}$ is the electron flux in unit of $10^{35}$ electrons $\mathrm{s}^{-1}$, and $\delta$ is the powerlaw index of nonthermal electrons (see Figure 12). The values of $E_{c}$ are 20, 30, 23, and $28 \mathrm{keV}$, respectively. Using Equation 4 and the above parameters, we estimated the total nonthermal energy in flare-accelerated electrons. The values of $E_{n t h}$, falling in the range of $(1.3-13) \times 10^{30} \mathrm{erg}$, are listed in the ninth column of Table 3. The ratio of $E_{n t h} / E_{t h, G}$ is between $\sim 2.4$ and $\sim 6.5$. It should be emphasized that these calculated values are lower limits of real nonthermal energies, which rely sensitively on $E_{c}$ (Warmuth and Mann, 2020). Besides, we did not consider the nonthermal energy in flare-accelerated ions. 

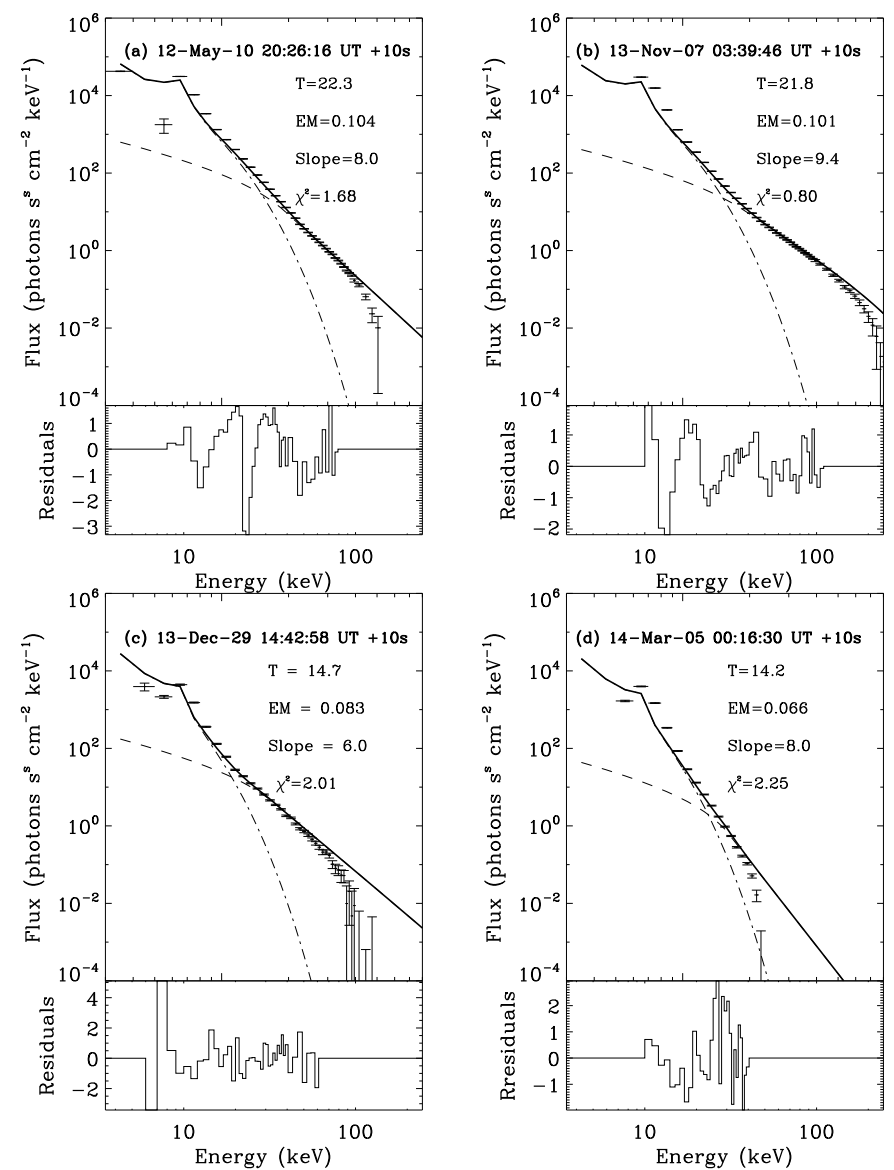

Figure 12. Selected HXR spectra of the four flares obtained from RHESSI observations and the corresponding normalized residuals of the spectral fitting. The observed data are represented by the points with error bars. The fitted thermal and nonthermal power-law components are drawn with dot-dashed lines and dashed lines, respectively. The sum of both components are drawn with thick solid lines. The fitted parameters, including $T$ in unit of MK, EM in unit of $10^{49} \mathrm{~cm}^{-3}$, and power-law index of electrons are labeled.

\subsection{Magnetic Free Energy}

As mentioned in Section 2, Figure 2 shows the vector magnetograms of the four ARs where flares took place. Both potential field and nonpotential field extrapolations were performed. Figure 3 shows the nonpotential magnetic field lines (blue lines) of the flares. The magnetic free energy $\left(E_{m a g}\right)$ is defined as the excess magnetic energy of the NLFFF $\left(E_{n p}\right)$ relative to the energy of potential field $\left(E_{p}\right)$ :

$$
E_{m a g}=E_{n p}-E_{p}=\int_{V} \frac{B_{n p}^{2}-B_{p}^{2}}{8 \pi} d V
$$


We calculated $E_{m a g}$ in the flare regions as enclosed by the red boxes in Figure2. The estimated $E_{\text {mag }}$, ranging from $1.8 \times 10^{30}$ to $1.7 \times 10^{31} \mathrm{erg}$, are listed in the tenth column of Table 3 . It is obvious that the free magnetic energies are larger than the nonthermal energies and radiative output in $1-370 \AA$, indicating that the accumulated free energy before flare is sufficient to provide the kinetic energy in flare-accelerated energies and radiation, thus validating the magnetic nature of confined flares (Priest and Forbes, 2002). The ratio of $E_{n t h} / E_{m a g}$ for CRFs falls in the range of $70 \%-76 \%$ (see the last column of Table 3), which is much higher than that of X-class eruptive flares (Feng et al., 2013; Thalmann et al., 2015). In other words, more free energy is converted into the kinetic energy of flare-accelerated electrons in confined flares than in eruptive flares, because a large fraction of free energy is converted into the kinetic, thermal, and potential energies of CMEs for eruptive flares (Reeves et al., 2010; Emslie et al., 2012; Feng et al., 2013).

\section{Discussion}

As mentioned in Section 11 Zhang et al. (2019) explored the energy partition in two M-class CRFs. The radiative outputs in $1-8 \AA$ and $1-70 \AA$ are obtained using the observations of GOES and SDO/EVE. Total radiative loss and peak thermal plasma are calculated using the observations of GOES and SDO/AIA. Nonthermal energy of electrons are derived using the observation of RHESSI (see their Table 2). The radiation in 70-370 $\AA$, total solar irradiance, nonthermal energy of ions, and dissipated magnetic free energy are estimated according to previous statistical works. In this study, we calculated the radiation in 70-370 $\AA$ using the observations of SDO/EVE and magnetic free energy using magnetic extrapolation based on the vector magnetograms from SDO/HMI. The results combining these six events with increasing flare importance (or peak GOES flux) are plotted in Figure 13. The orders of magnitude of the energy components are clearly demonstrated. Moreover, for each component, the values increase systematically with flare importance, suggesting that more energy is involved in larger flares. Our findings are in accordance with previous statistical results (Warmuth and Mann, 2020).

In Figure 14, the left panel shows the scatter plot of the six events. The relationship between the nonthermal and thermal energies is illustrated with cyan circles, while the relationship between the nonthermal energy and heating requirement (including the thermal energy and radiative loss) is illustrated with magenta circles. There is a good linear correlation between the nonthermal energy and thermal energy, validating the previous results for nine mediumsized flares (Saint-Hilaire and Benz, 2005). It is obvious that the nonthermal energies are higher than the heating requirements of hot plasma, at least for the six events we have studied. Kushwaha et al. (2015) investigated an M6.2 confined flare on 2004 July 14 . The peak thermal energy and nonthermal energy are calculated to be $3.89 \times 10^{29} \mathrm{erg}$ and $3.03 \times 10^{30} \mathrm{erg}$, respectively. Hence, the ratio of $E_{n t h} / E_{t h}$ reaches $\sim 7.5$, which is consistent with the present work. 


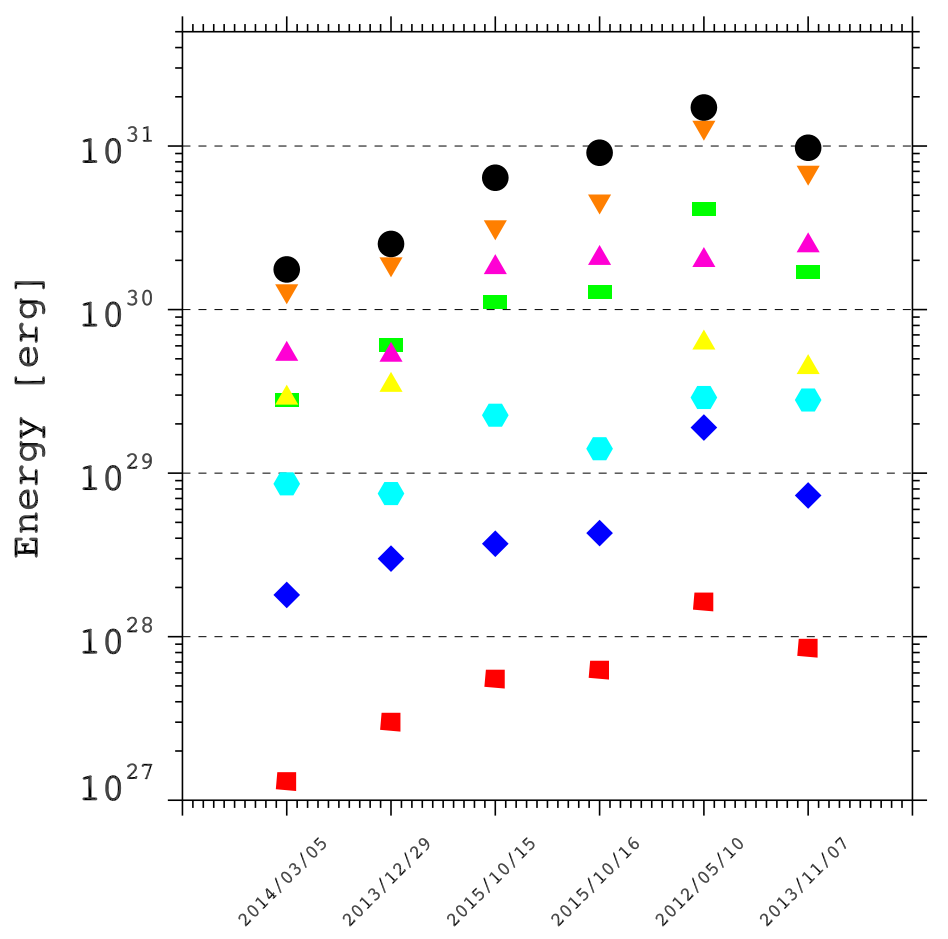

Figure 13. Energy components of the four events in this study and the previous two events in Zhang et al. (2019). The six events are arranged with increasing flare importance (see text for detail). The radiative outputs in $1-8 \AA, 1-70 \AA, 70-370 \AA$, total radiative loss, peak thermal energy derived from GOES and RHESSI, nonthermal energy in electrons, and magnetic free energy are labeled with red squares, green rectangles, blue diamonds, cyan hexagons, magenta triangles, yellow triangles, orange triangles, and black circles, respectively.

The right panel of Figure 14 shows the scatter plot of the six events to illustrate the relationship between the maximal temperatures of GOES $\left(T_{G}\right.$ in MK) and RHESSI ( $T_{R}$ in MK). It is seen that $T_{R}$ is higher than $T_{G}$ in most cases and a good linear correlation exists between the two parameters. A linear fit yields $T_{R}=1.65 T_{G}-5.85$, which lies between $T_{R}=1.12 T_{G}-3.12$ (Battaglia, Grigis, and Benz, 2005) and $T_{R}=1.78 T_{G}-4.61$ (Warmuth and Mann, 2016a). It is noted that our study has limitations due to the small sample size. Additional statistical studies using more events and numerical simulations are worthwhile to draw a decisive conclusion.

\section{Summary}

In this paper, we investigated the energy partition of four confined circularribbon flares near the solar disk center. Using multiwavelength observations from SDO, GOES, and RHESSI, we calculated different energy components, including the radiative outputs in $1-8,1-70$, and $70-370 \AA$, total radiative loss, peak 

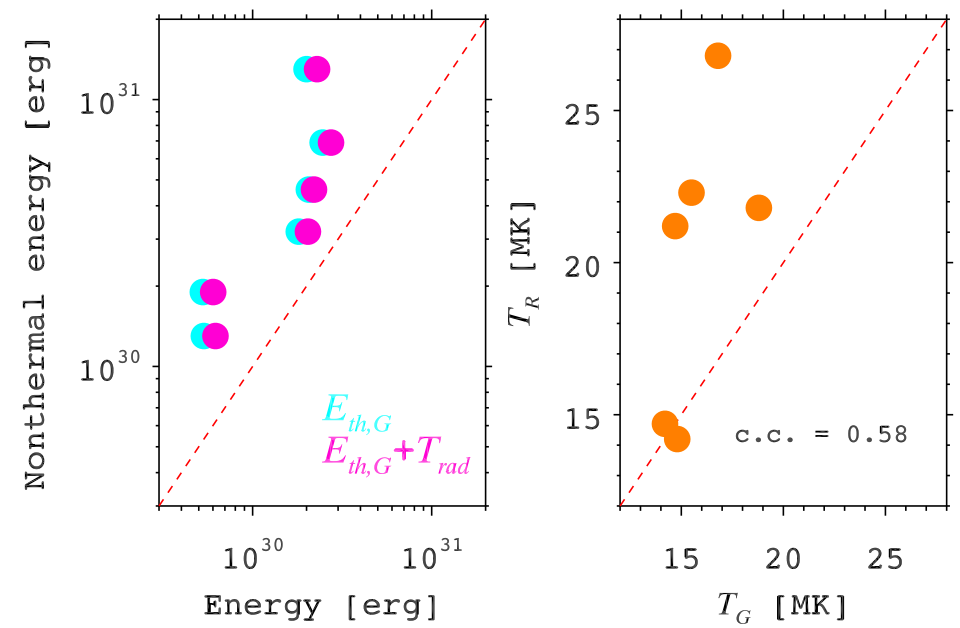

Figure 14. Left panel: scatter plot showing the relationship between nonthermal energy and thermal energy (cyan circles) and heating requirement (magenta circles). Right panel: scatter plot showing the relationship between the maximal temperatures of GOES $\left(T_{G}\right)$ and RHESSI $\left(T_{R}\right)$. The correlation coefficient $(\sim 0.58)$ is labeled. The red dashed lines in both panels represent the $y=x$ curves.

thermal energy derived from GOES and RHESSI, nonthermal energy in flareaccelerated electrons, and magnetic free energy before flares. The main results are as follows:

1. The energy components increase systematically with the flare importance or peak GOES flux, indicating that more energies are involved in larger flares. The magnetic free energies are larger than the nonthermal energies and radiative outputs of flares, which is consistent with the magnetic nature of flares. The ratio $\frac{E_{n t h}}{E_{\text {mag }}}$ of the four flares, being $0.70-0.76$, is considerably higher than that of eruptive flares. Hence, this ratio may serve as an important factor that discriminates confined and eruptive flares. The nonthermal energies are sufficient to provide the heating requirements including the peak thermal energy and radiative loss.

2. Our findings impose constraint on theoretical models of confined CRFs and have potential implication for the space weather forecast. Statistical studies based on a larger sample are especially needed to draw decisive conclusions.

Acknowledgements The authors are grateful to the referee for valuable suggestions. The authors thank Drs. Tie Liu, Yanjie, Liu, and Ya Wang for helpful discussion. SDO is a mission of NASA's Living With a Star Program. AIA and HMI data are courtesy of the NASA/SDO science teams. This work is funded by NSFC grants (No. 11790302, 11773079, 41761134088, 11473071), the International Cooperation and Interchange Program (11961131002), the Youth Innovation Promotion Association CAS, CAS Key Laboratory of Solar Activity, National Astronomical Observatories (KLSA202006), and the Strategic Priority Research Program on Space Science, CAS (XDA15052200, XDA15320301). 


\section{References}

Amari, T., Luciani, J.F., Mikic, Z., and Linker, J.: 2000, The Astrophysical Journal 529, L49. Antiochos, S.K., DeVore, C.R., and Klimchuk, J.A.: 1999, The Astrophysical Journal 510, 485.

Aschwanden, M.J., Xu, Y., and Jing, J.: 2014, The Astrophysical Journal 797, 50.

Aschwanden, M.J., Boerner, P., Ryan, D., Caspi, A., McTiernan, J.M., and Warren, H.P.: 2015, The Astrophysical Journal 802, 53.

Aschwanden, M.J., Holman, G., O'Flannagain, A., Caspi, A., McTiernan, J.M., and Kontar, E.P.: 2016, The Astrophysical Journal 832, 27.

Aschwanden, M.J., Caspi, A., Cohen, C.M.S., Holman, G., Jing, J., Kretzschmar, M., and, ...: 2017, The Astrophysical Journal 836, 17.

Battaglia, M., Grigis, P.C., and Benz, A.O.: 2005, Astronomy and Astrophysics 439, 737.

Caspi, A., Krucker, S., and Lin, R.P.: 2014, The Astrophysical Journal 781, 43.

Chen, P. F. \& Shibata, K. 2000, Astrophys. J., 545, 524.

Cheng, X., Zhang, J., Ding, M.D., Guo, Y., and Su, J.T.: 2011, The Astrophysical Journal 732, 87. doi:10.1088/0004-637X/732/2/87.

Chiu, Y.T. and Hilton, H.H.: 1977, The Astrophysical Journal 212, 873. doi:10.1086/155111.

Cox, D.P., and Tucker, W.H.: 1969, The Astrophysical Journal 157, 1157.

Dai, J., Zhang, Q.M., Su, Y.N., and Ji, H.S.: 2021, Astronomy and Astrophysics 646, A12.

Dere, K.P., Del Zanna, G., Young, P.R., Landi, E., and Sutherland, R.S.: 2019, The Astrophysical Journal Supplement Series 241, 22. doi:10.3847/1538-4365/ab05cf.

Devi, P., Joshi, B., Chandra, R., Mitra, P.K., Veronig, A.M., and Joshi, R.: 2020, Solar Physics 295, 75. doi:10.1007/s11207-020-01642-y.

Emslie, A.G., Kucharek, H., Dennis, B.R., Gopalswamy, N., Holman, G.D., Share, G.H., Vourlidas, A., Forbes, T.G., Gallagher, P.T., Mason, G.M., Metcalf, T.R., Mewaldt, R.A., Murphy, R.J., and, ...: 2004, Journal of Geophysical Research (Space Physics) 109, A10104.

Emslie, A.G., Dennis, B.R., Holman, G.D., and Hudson, H.S.: 2005, Journal of Geophysical Research (Space Physics) 110, A11103.

Emslie, A.G., Dennis, B.R., Shih, A.Y., Chamberlin, P.C., Mewaldt, R.A., Moore, C.S., Share, G.H., Vourlidas, A., and Welsch, B.T.: 2012, The Astrophysical Journal 759, 71.

Feng, L., Wiegelmann, T., Su, Y., Inhester, B., Li, Y.P., Sun, X.D., and Gan, W.Q.: 2013, The Astrophysical Journal 765, 37.

Fletcher, L., Dennis, B.R., Hudson, H.S., Krucker, S., Phillips, K., Veronig, A., and, ...: 2011, Space Science Reviews 159, 19.

Forbes, T.G. and Acton, L.W.: 1996, The Astrophysical Journal 459, 330

Gopalswamy, N.: 2016, Geoscience Letters 3, 8.

Hernandez-Perez, A., Thalmann, J.K., Veronig, A.M., Su, Y., Gömöry, P., and Dickson, E.C.: 2017, The Astrophysical Journal 847, 124.

Hou, Y., Li, T., Yang, S., and Zhang, J.: 2019, The Astrophysical Journal 871, 4. doi:10.3847/1538-4357/aaf4f4.

Hurford, G.J., Schmahl, E.J., Schwartz, R.A., Conway, A.J., Aschwanden, M.J., Csillaghy, A., and, ...: 2002, Solar Physics 210, 61. doi:10.1023/A:1022436213688.

Inglis, A.R. and Christe, S.: 2014, The Astrophysical Journal 789, 116. doi:10.1088/0004$637 \mathrm{X} / 789 / 2 / 116$

Ji, H., Wang, H., Schmahl, E.J., Moon, Y.-J., and Jiang, Y.: 2003, The Astrophysical Journal 595, L135.

Joshi, N.C., Liu, C., Sun, X., Wang, H., Magara, T., and Moon, Y.-J.: 2015, The Astrophysical Journal 812, 50. doi:10.1088/0004-637X/812/1/50.

Joshi, N.C., Joshi, B., and Mitra, P.K.: 2021, Monthly Notices of the Royal Astronomical Society 501, 4703. doi:10.1093/mnras/staa3480.

Kashapova, L.K., Kupriyanova, E.G., Xu, Z., Reid, H.A.S., and Kolotkov, D.Y.: 2020, Astronomy and Astrophysics 642, A195. doi:10.1051/0004-6361/201833947.

Kliem, B., Lee, J., Liu, R., White, S.M., Liu, C., and Masuda, S.: 2021, arXiv e-prints, arXiv:2101.02181.

Kretzschmar, M., de Wit, T.D., Schmutz, W., Mekaoui, S., Hochedez, J.-F., and Dewitte, S.: 2010, Nature Physics 6, 690. doi:10.1038/nphys1741.

Kushwaha, U., Joshi, B., Cho, K.-S., Veronig, A., Tiwari, S.K., and Mathew, S.K.: 2014, The Astrophysical Journal 791, 23. doi:10.1088/0004-637X/791/1/23.

Kushwaha, U., Joshi, B., Veronig, A.M., and Moon, Y.-J.: 2015, The Astrophysical Journal 807, 101. doi:10.1088/0004-637X/807/1/101. 
Lemen, J.R., Title, A.M., Akin, D.J., Boerner, P.F., Chou, C., Drake, J.F., and, ...: 2012, Solar Physics 275, 17.

Lee, K.-S., Hara, H., Watanabe, K., Joshi, A.D., Brooks, D.H., Imada, S., and, ...: 2020, The Astrophysical Journal 895, 42. doi:10.3847/1538-4357/ab8bce.

Li, H., Jiang, Y., Yang, J., Yang, B., Xu, Z., Hong, J., and, ...: 2017, The Astrophysical Journal 836, 235.

Li, T., Hou, Y., Yang, S., Zhang, J., Liu, L., and Veronig, A.M.: 2020, The Astrophysical Journal 900, 128. doi:10.3847/1538-4357/aba6ef.

Lin, R.P., Dennis, B.R., Hurford, G.J., Smith, D.M., Zehnder, A., Harvey, P.R., and, ...: 2002, Solar Physics 210, 3.

Lin, J. and Forbes, T.G.: 2000, Journal of Geophysical Research 105, 2375.

Liu, R., Titov, V.S., Gou, T., Wang, Y., Liu, K., and Wang, H.: 2014, The Astrophysical Journal 790, 8

Liu, C., Deng, N., Liu, R., Lee, J., Pariat, É., Wiegelmann, T., and, ...: 2015, The Astrophysical Journal 812, L19.

Liu, C., Prasad, A., Lee, J., and Wang, H.: 2020, The Astrophysical Journal 899, 34.

Masson, S., Pariat, E., Aulanier, G., and Schrijver, C.J.: 2009, The Astrophysical Journal 700, 559.

Milligan, R.O., Chamberlin, P.C., Hudson, H.S., Woods, T.N., Mathioudakis, M., Fletcher, L., and, ...: 2012, The Astrophysical Journal 748, L14. doi:10.1088/2041-8205/748/1/L14.

Milligan, R.O., Kerr, G.S., Dennis, B.R., Hudson, H.S., Fletcher, L., Allred, J.C., and, ...: 2014, The Astrophysical Journal 793, 70. doi:10.1088/0004-637X/793/2/70.

Moore, R.L., Sterling, A.C., Hudson, H.S., and Lemen, J.R.: 2001, The Astrophysical Journal 552, 833 .

Ning, Z., and Cao, W.: 2010, Solar Physics 264, 329.

Ning, H., Chen, Y., Wu, Z., Su, Y., Tian, H., Li, G., and, ...: 2018, The Astrophysical Journal 854, 178 .

Patsourakos, S., Vourlidas, A., Török, T., Kliem, B., Antiochos, S.K., Archontis, V., and, ...: 2020, Space Science Reviews 216, 131. doi:10.1007/s11214-020-00757-9.

Prasad, A., Dissauer, K., Hu, Q., Bhattacharyya, R., Veronig, A.M., Kumar, S., and, ...: 2020, The Astrophysical Journal 903, 129. doi:10.3847/1538-4357/abb8d2.

Priest, E.R. and Forbes, T.G.: 2002, Astronomy and Astrophysics Review 10, 313.

Reeves, K.K., Linker, J.A., Mikić, Z., and Forbes, T.G.: 2010, The Astrophysical Journal 721, 1547. doi:10.1088/0004-637X/721/2/1547.

Saint-Hilaire, P. and Benz, A.O.: 2005, Astronomy and Astrophysics 435, 743.

Scherrer, P.H., Schou, J., Bush, R.I., Kosovichev, A.G., Bogart, R.S., Hoeksema, J.T., and, ...: 2012, Solar Physics 275, 207.

Seehafer, N.: 1978, Solar Physics 58, 215. doi:10.1007/BF00157267.

Shimojo, M. and Shibata, K.: 2000, The Astrophysical Journal 542, 1100.

Song, Y. and Tian, H.: 2018, The Astrophysical Journal 867, 159

Stoiser, S., Veronig, A.M., Aurass, H., and Hanslmeier, A.: 2007, Solar Physics 246, 339. doi:10.1007/s11207-007-9066-7.

Su, Y., Surges, V., van Ballegooijen, A., DeLuca, E., and Golub, L.: 2011, The Astrophysical Journal 734, 53.

Su, Y., Veronig, A.M., Holman, G.D., Dennis, B.R., Wang, T., Temmer, M., and, ...: 2013, Nature Physics 9, 489.

Su, Y., van Ballegooijen, A., McCauley, P., Ji, H., Reeves, K.K., and DeLuca, E.E.: 2015, The Astrophysical Journal 807, 144.

Sun, X., Hoeksema, J.T., Liu, Y., Aulanier, G., Su, Y., Hannah, I.G., and Hock, R.A.: 2013, The Astrophysical Journal 778, 139

Sun, X., Bobra, M.G., Hoeksema, J.T., Liu, Y., Li, Y., Shen, C., and, ...: 2015, The Astrophysical Journal 804, L28.

Thalmann, J.K., Su, Y., Temmer, M., and Veronig, A.M.: 2015, The Astrophysical Journal 801, L23.

Veronig, A.M. and Polanec, W.: 2015, Solar Physics 290, 2923.

Wang, H. and Liu, C.: 2012, The Astrophysical Journal 760, 101.

Warmuth, A. and Mann, G.: 2016, Astronomy and Astrophysics 588, A115.

Warmuth, A. and Mann, G.: 2016, Astronomy and Astrophysics 588, A116.

Warmuth, A. and Mann, G.: 2020, Astronomy and Astrophysics 644, A172. doi:10.1051/0004$6361 / 202039529$.

Webb, D.F., and Howard, T.A.: 2012, Living Reviews in Solar Physics 9, 3. 
White, S.M., Thomas, R.J., and Schwartz, R.A.: 2005, Solar Physics 227, 231.

Wiegelmann, T., Inhester, B., and Sakurai, T.: 2006, Solar Physics 233, 215.

Wiegelmann, T., Thalmann, J.K., Schrijver, C.J., De Rosa, M.L., and Metcalf, T.R.: 2008, Solar Physics 247, 249.

Woods, T.N., Eparvier, F.G., Hock, R., Jones, A.R., Woodraska, D., Judge, D., and, ...: 2012, Solar Physics 275, 115.

Xu, Z., Yang, K., Guo, Y., Zhao, J., Zhao, Z.J., and Kashapova, L.: 2017, The Astrophysical Journal 851, 30.

Yan, X., Xue, Z., Cheng, X., Zhang, J., Wang, J., Kong, D., and, ...: 2020, The Astrophysical Journal 889, 106. doi:10.3847/1538-4357/ab61f3.

Yang, S. and Zhang, J.: 2018, The Astrophysical Journal 860, L25.

Yang, S., Zhang, Q., Xu, Z., Zhang, J., Zhong, Z., and Guo, Y.: 2020, The Astrophysical Journal, 898, 101.

Zhang, Q.M., Chen, P.F., Guo, Y., Fang, C., and Ding, M.D.: 2012, The Astrophysical Journal 746, 19. doi:10.1088/0004-637X/746/1/19.

Zhang, Q.M., Ning, Z.J., Guo, Y., Zhou, T.H., Cheng, X., Ji, H.S., and, ...: 2015, The Astrophysical Journal 805, 4.

Zhang, Q.M., Li, D., Ning, Z.J., Su, Y.N., Ji, H.S., and Guo, Y.: 2016, The Astrophysical Journal 827, 27.

Zhang, Q.M., Li, D., and Ning, Z.J.: 2016, The Astrophysical Journal 832, 65.

Zhang, Q.M., Li, D., and Huang, Y.: 2019, The Astrophysical Journal 870, 109.

Zhang, Q.M., Cheng, J.X., Feng, L., Su, Y., Lu, L., Huang, Y., Li, D., Zhou, T.H., and Chen, J.L.: 2019, The Astrophysical Journal 883, 124.

Zhang, Q.M., Yang, S.H., Li, T., Hou, Y.J., and Li, Y.: 2020, Astronomy and Astrophysics 636, L11.

Zhang, Q.M., Huang, Z.H., Hou, Y.J., Li, D., Ning, Z.J., and Wu, Z.: 2021, arXiv e-prints, arXiv:2101.06629. 